Article

\title{
Dose Intensive Rituximab and High-Dose Methylprednisolone in Elderly or Unfit Patients with Relapsed Chronic Lymphocytic Leukemia
}

\author{
Regina Pileckyte ${ }^{1,2, *}$, Vilma Valceckiene ${ }^{1}$, Mindaugas Stoskus ${ }^{1}$, Reda Matuzeviciene ${ }^{3}$, \\ Jurgita Sejoniene ${ }^{4}$, Tadas Zvirblis ${ }^{1}$ and Laimonas Griskevicius ${ }^{1,2}$ \\ 1 Hematology, Oncology and Transfusion Medicine Center, Vilnius University Hospital Santaros Klinikos, \\ 08661 Vilnius, Lithuania; vilma.valceckiene@santa.lt (V.V.); mindaugas.stoskus@santa.lt (M.S.); \\ tadas.zvirblis@gmail.com (T.Z.); laimonas.griskevicius@santa.lt (L.G.) \\ 2 Institute of Clinical Medicine, Vilnius University, 08661 Vilnius, Lithuania \\ 3 Laboratory Diagnostics Centre, Vilnius University Hospital Santaros Klinikos, 08661 Vilnius, Lithuania; \\ reda.matuzeviciene@santa.lt \\ 4 Radiology and Nuclear Medicine Center, Vilnius University Hospital Santaros Klinikos, 08661 Vilnius, \\ Lithuania; jurgita.sejoniene@santa.lt \\ * Correspondence: regina.pileckyte@santa.lt; Tel.: +370-5236-529; Fax: +370-5236-5088
}

Received: 12 August 2019; Accepted: 25 October 2019; Published: 29 October 2019

\begin{abstract}
Background and Objectives: BTK and BCL2 inhibitors have changed the treatment paradigms of high-risk and elderly patients with chronic lymphocytic leukemia (CLL), but their long-term efficacy and toxicity are still unknown and the costs are considerable. Our previous data showed that Rituximab (Rtx) and high-dose methylprednisolone (HDMP) can be an effective and safe treatment option for relapsed high-risk CLL patients. Materials and Methods: We explored the efficacy and safety of a higher Rtx dose in combination with a shorter (3-day) schedule of HDMP in relapsed elderly or unfit CLL patients. Results: Twenty-five patients were included in the phase-two, single-arm trial. The median progression free survival (PFS) was 11 months (range 10-12). Median OS was 68 (range 47-89) months. Adverse events (AE) were mainly grade I-II ${ }^{\circ}(77 \%)$ and no deaths occurred during the treatment period. Conclusions: 3-day HDMP and Rtx was associated with clinically meaningful improvement in most patients. The median PFS in 3-day and 5-day HDMP studies was similar and the toxicity of the 3-day HDMP schedule proved to be lower. The HDMP and Rtx combination can still be applied in some relapsed high-risk and elderly or unfit CLL patients if new targeted therapies are contraindicated or unavailable. (ClinicalTrials.gov identifier: NCT01576588).
\end{abstract}

Keywords: chronic lymphocytic leukemia; rituximab; high-dose methylprednisolone; elderly

\section{Introduction}

Chronic lymphocytic leukemia (CLL) is the most prevalent type of leukemia among adults [1]. The majority of patients are older than 70 and have coexisting conditions.

Before the era of targeted therapy, standard treatments included combinations of purine analogues, alkylating agents, and monoclonal antibodies. Chemoimmunotherapy can provide long-term disease control in younger patients without major comorbidities, but may have unacceptable side effects in older patients in whom non-myelotoxic approaches may be preferred.

New agents such as BTK and BCL2 inhibitors have changed the treatment paradigms of high-risk and/or elderly patients with CLL [1,2], but the long-term efficacies and toxicities are still unknown and the costs are considerable. 
Rituximab (Rtx) and high-dose methylprednisolone (HDMP) can be an effective and safe treatment option for relapsed CLL in different settings [3-5]. Earlier studies [4,5] have explored the combination of HDMP with the anti-CD20 antibody Rtx in relapsed and fludarabine-refractory CLL patients using various Rtx dosing schedules and treatment durations. Our previous study of HDMP and Rtx combination in relapsed high-risk CLL, including TP53 dysfunction, demonstrated efficacy with an overall response rate (ORR) of $62 \%$ [3]. However, there were three deaths throughout the study. In order to reduce toxicity, we have attempted to evaluate the combination of a shorter HDMP schedule and an Rtx dose that is higher than the standard dosage. Here, we present the results of the HDMP and Rtx combination given to elderly, pretreated patients.

\section{Materials and Methods}

\subsection{Patients}

A phase II, single-arm, open-label, prospective study was conducted to evaluate HDMP in combination with Rtx in elderly or unfit patients with relapsed CLL.

Eligible patients had CD20+ CLL with treatment indications according to the criteria of the International Workshop on Chronic Lymphocytic Leukemia (IWCLL 2008) [6]. They had relapsed or progressive disease symptoms after at least one prior chemotherapy regimen, and were 18-64 years of age with comorbidities (cumulative illness rating scale (CIRS) $>6$ ) or $\geq 65$ years of age irrespective of comorbidities. Exclusion criteria included intolerance to exogenous protein administration or previous reaction to Rtx treatment, active infections, tuberculosis or fungal infections within the past six months, active peptic ulcers, severe organ deficiency preventing participation in the study, inadequately controlled diabetes mellitus, allergic disorders in need of chronic glucocorticoid therapy, and pregnancy or lactation.

\subsection{Assessments and End Points}

Pretreatment evaluation consisted of laboratory tests, a bone marrow biopsy and aspiration with immunophenotyping, and a neck, chest, abdominal, and pelvic computer tomography (CT). The coding region of the TP53 gene (exons 2-11) was PCR-amplified and scanned for mutations by high-resolution fluorescent melting (HRM) curve analysis as previously described [7]. Mutations were confirmed by direct sequencing. Immunoglobulin heavy-chain variable region $(I g H V)$ mutational status was determined by direct DNA sequencing [8]. The sequences were aligned to IgHV sequences from the IMGT database. Gene sequences deviating more than $2 \%$ from the corresponding germline gene were defined as mutated. The presence of $17 \mathrm{p}$ deletion, 11q deletion, trisomy 12 , and 13q deletion was assessed by FISH analysis using commercially available probes (Kreatech Diagnostics, Amsterdam, The Netherlands). ZAP70 expression in microbeads purified CD19+ cells (purity over 99\%) was analyzed using RT-qPCR. The cut-off value for ZAP70 expression was determined using CD19+ cells from 30 healthy donors. CIRS assessment was performed according to guidelines [9]. Adverse events were reported according to the National Cancer Institute Common Toxicity Criteria (version 4.0) [10], and hematological toxicity was evaluated according to IWCLL 2008 guidelines [6].

The response to therapy was confirmed by CT two to three months following the end of treatment, and complete responses were confirmed by bone marrow biopsy. Minimal residual disease (MRD) was analyzed according to international guidelines [6]. During the follow-up period, patients underwent physical and laboratory tests every three months until disease progression ceased or death occurred.

The primary objective was to determine the treatment response rate. Secondary objectives were used to determine progression-free survival (PFS), overall survival (OS), and the safety profile of Rtx and HDMP. The study protocol was approved by the Lithuanian Bioethics Committee and the study was conducted according to the Declaration of Helsinki. The ethical code number is P-11-005 and the date of approval was 13-01-2011. All patients provided written informed consent. 


\subsection{Study Treatment and Monitoring}

HDMP was administered at a daily dose of $1 \mathrm{~g} / \mathrm{m}^{2}$ intravenously over $4 \mathrm{~h}$ for three consecutive days for four cycles. After 14 patients had been included, the protocol was amended to allow an additional two HDMP cycles to be given to patients without significant toxicity. Rtx was administered at a dose of $1000 \mathrm{mg} / \mathrm{m}^{2}$, following HDMP infusion for 4 courses. To decrease the incidence of initial infusion reactions, patients received the first dose of Rtx split into $50 \mathrm{mg}$ on day one, $150 \mathrm{mg}$ on day two, and the remaining $800 \mathrm{mg}$ on day three. A full dose was given on the first day during $2-4$ courses and the regimen was repeated every 21 days. There were no dose adjustments for Rtx. If non-hematological clinically significant grade III-IV toxicities related to glucocorticoid occurred, the HDMP dose could be decreased by $50 \%$ during subsequent doses.

\subsection{Statistical Methods}

Statistical analysis of survival rates and responses according to IWCLL 2008 guidelines were performed on an intent-to-treat basis for all enrolled patients. Adverse events (AEs) and clinical safety data were summarized using descriptive statistics. Response to treatment was expressed as the proportion of patients who achieved at least a partial response (PR). Paired Student's t-test was used to compare blood count values during treatment. Survival trends were evaluated using the Kaplan-Meier method. Cox regression analysis was used to evaluate the impact of different prognostic factors on PFS and OS. Two-tailed $p$ values $<0.05$ were considered significant. Statistical analysis was performed using SAS version 9.2. RP, TZ, and LG analyzed the data. All authors had access to primary clinical trial data.

\subsection{Comparison of LT-CLL-001 and LT-CLL-2s Study Results}

Indirect comparison of treatment schedule, prognostic factors, PFS, OS, and salvage therapy were described.

\section{Results}

\subsection{Patient Characteristics}

Between October 2011 and October 2014, 27 patients were screened, with 25 patients being included in the study. Two patients were screen failures: one due to active hepatitis $B$ and another because of concomitant myelodysplastic syndrome. Patient demographics and baseline characteristics are summarized in Table 1. The median age was 73 years (range 65-80), the CIRS median was five (range 1-8), patients had received a median of two (1-6) previous treatment lines, $80 \%$ of the patients suffered from B symptoms, five (20\%) had 17p deletion and/or TP53 mutation, three (12\%) had 11q deletion, 17 (68\%) had unmutated $I g V H, 16(64 \%)$ had hyperexpression of ZAP70, and $96 \%$ had elevated $\beta_{2}$ microglobulin $>3.5 \mathrm{mg} / \mathrm{L}$.

Sixteen patients were given $\leq 4$ courses (one stopped treatment after two courses due to disease progression, the remaining patients received the planned four courses). After protocol amendment, nine patients received $>4$ courses (eight patients were given six planned courses, and one patient received five courses due to an adverse event). 
Table 1. Patient baseline characteristics.

\begin{tabular}{|c|c|c|}
\hline Characteristic & $\mathbf{N}^{\circ}$ of Patients & $\%$ \\
\hline Number of patients & 25 & 100 \\
\hline Median age, years (range) & $73(65-80)$ & \\
\hline$\geq 75$ years & 9 & 36 \\
\hline \multicolumn{3}{|l|}{ Gender } \\
\hline Male & 7 & 28 \\
\hline Female & 18 & 72 \\
\hline CIRS median (range) & $5(1-8)$ & \\
\hline Median creatinine clearance (mL/min) & 54 & \\
\hline \multicolumn{3}{|l|}{ Rai stage } \\
\hline I-II & 10 & 40 \\
\hline III-IV & 15 & 60 \\
\hline Bulky lymphadenopathy (>5 cm) & 12 & 48 \\
\hline B symptoms & 20 & 80 \\
\hline CD20 expression $>80 \%$ & 18 & 72 \\
\hline B2 mikroglobulin $>3.5 \mathrm{mg} / \mathrm{L}$ * & 23 & 96 \\
\hline ZAP 70 possitive & 16 & 64 \\
\hline$I g H V$ unmutated & 17 & 68 \\
\hline \multicolumn{3}{|l|}{ Genetics } \\
\hline 17p deletion and/or TP53 deletion ** & 5 & 20 \\
\hline $11 q$ deletion only ${ }^{* * *}$ & 3 & 12 \\
\hline Trisomy 12 only & 2 & 8 \\
\hline $13 q$ deletion only & 7 & 28 \\
\hline Median no. of prior treatments (range) & $2(1-3)$ & \\
\hline Median time from the last treatment, months (range) & $20(0-153)$ & \\
\hline Fludarabine containing regimen & 7 & 28 \\
\hline Rituximab containing regimen & 3 & 10 \\
\hline
\end{tabular}

* 24 patients tested for B2 microglobulin ** One patient had 11q and 13q gene deletion, and one additional patient had a trisomy 12 deletion. ${ }^{* * *}$ One patient also had $13 q$ gene deletion.

\subsection{Response and Survival}

Overall response rate was $28 \%$ and all were partial responses, mainly due to residual lymphadenopathy. No MRD negative cases were confirmed. All 20 patients with B symptoms had their symptoms resolved. Significant improvement in anemia $(p<0.001)$ and reduction of lymphocytosis $(p<0.001)$ were noted (Figure 1). No significant change in neutrophil and platelet counts were observed.

The median follow-up for all patients was 50 (11-74) months, and 57 (range 41-74) months for the 12 patients who are still alive. The PFS was 11 months (range 10-12) (Figure 2). Median OS was 68 (range 47-89) months (Figure 3). No differences in response, PFS $(p=0.888)$, and OS $(p=0.152)$ were noted between the patients who received $\leq 4$ or $>4$ treatment courses. Three-year PFS was $5 \%$ and OS was $64 \%$.

Twelve (44\%) patients were alive at the last evaluation. Two patients failed to attend follow up and another 11 died. All patients progressed, but only one patient without indication for salvage treatment. One patient died due to complicated cholecystitis one year after study treatment completion and prior to salvage therapy. Other causes of death included disease progression in three patients, pre-existing cardiovascular disorders in two patients, and infections after salvage treatment in four patients. The cause of one patient's death is unknown. 

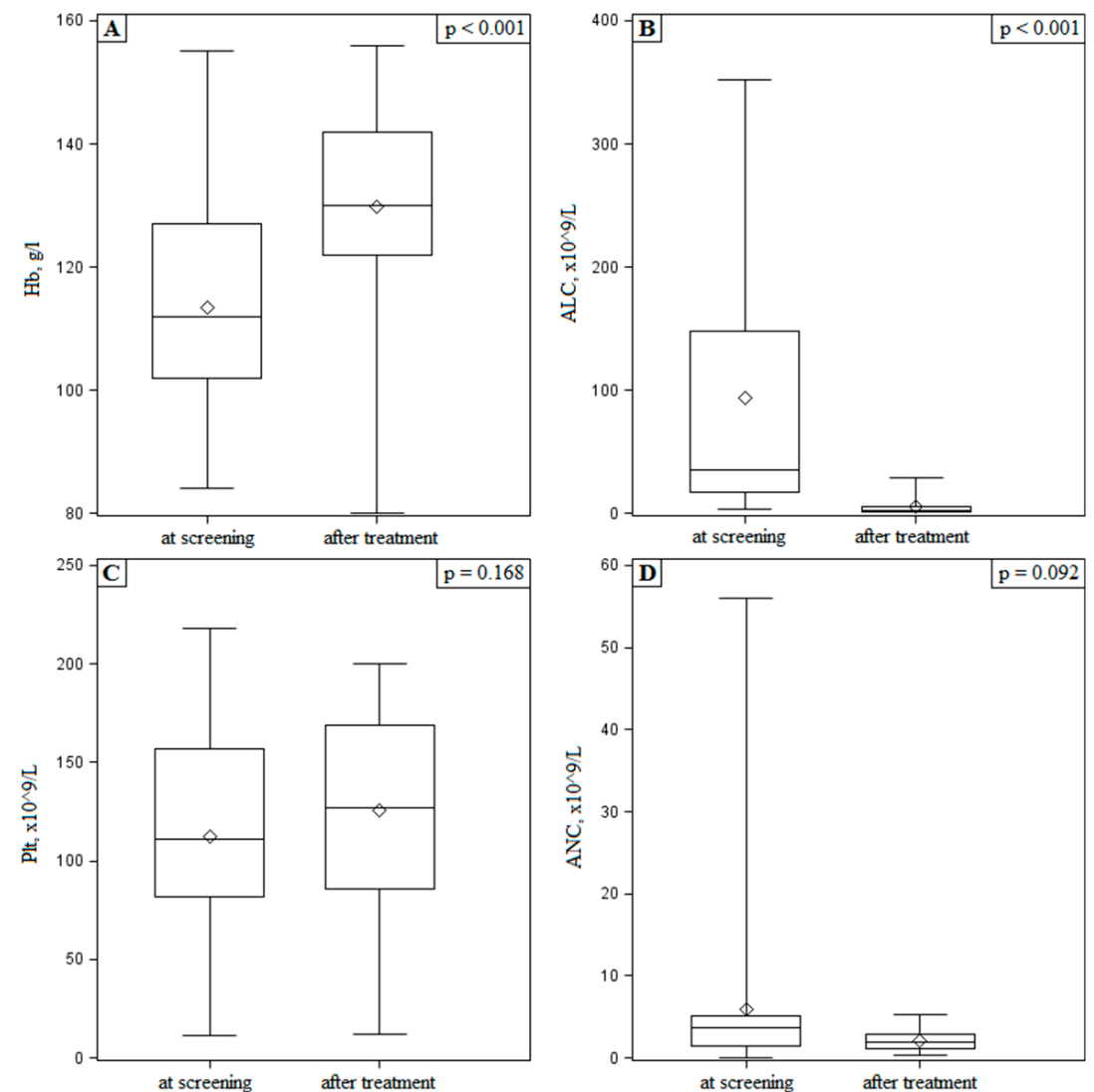

Figure 1. (A)-Hemoglobin ( $\mathrm{Hb})$; (B)-absolute lymphocyte count (ALC); (C)—platelet (Plt); (D)_absolute neutrophil count (ANC) at screening and cessation of treatment.

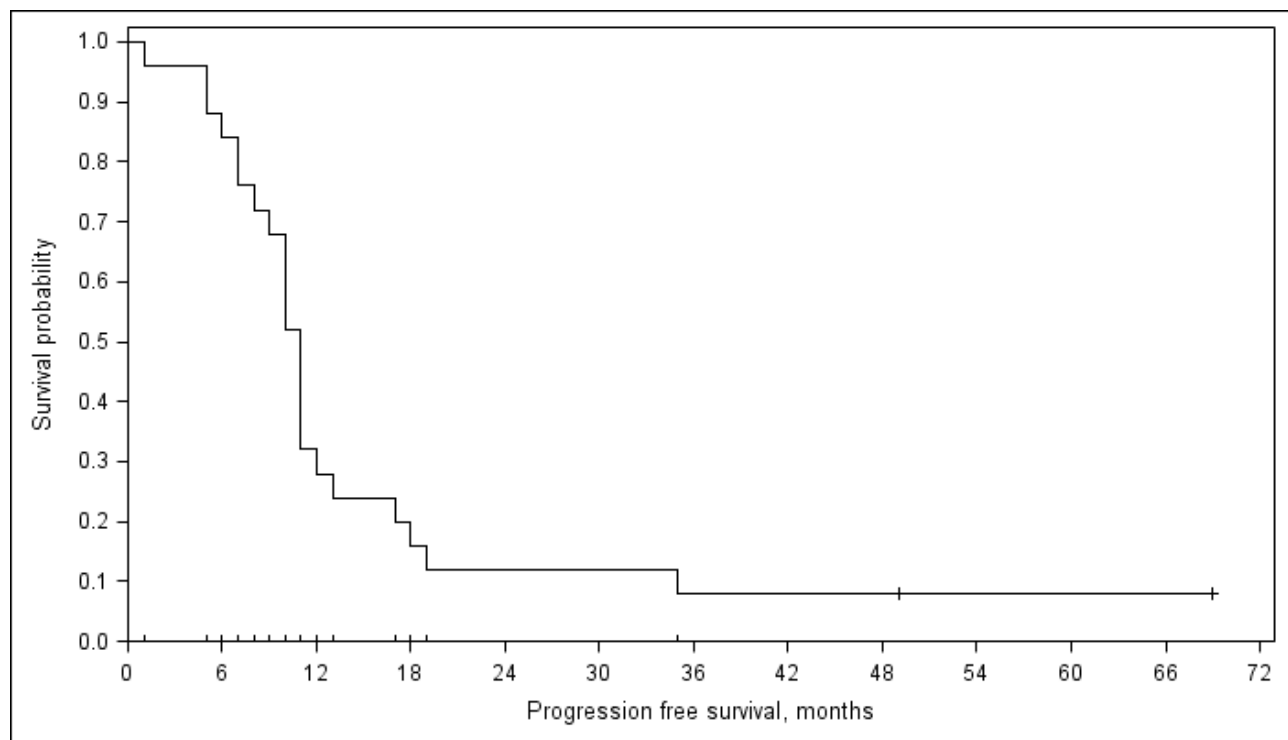

Figure 2. Progression-free survival (PFS). 


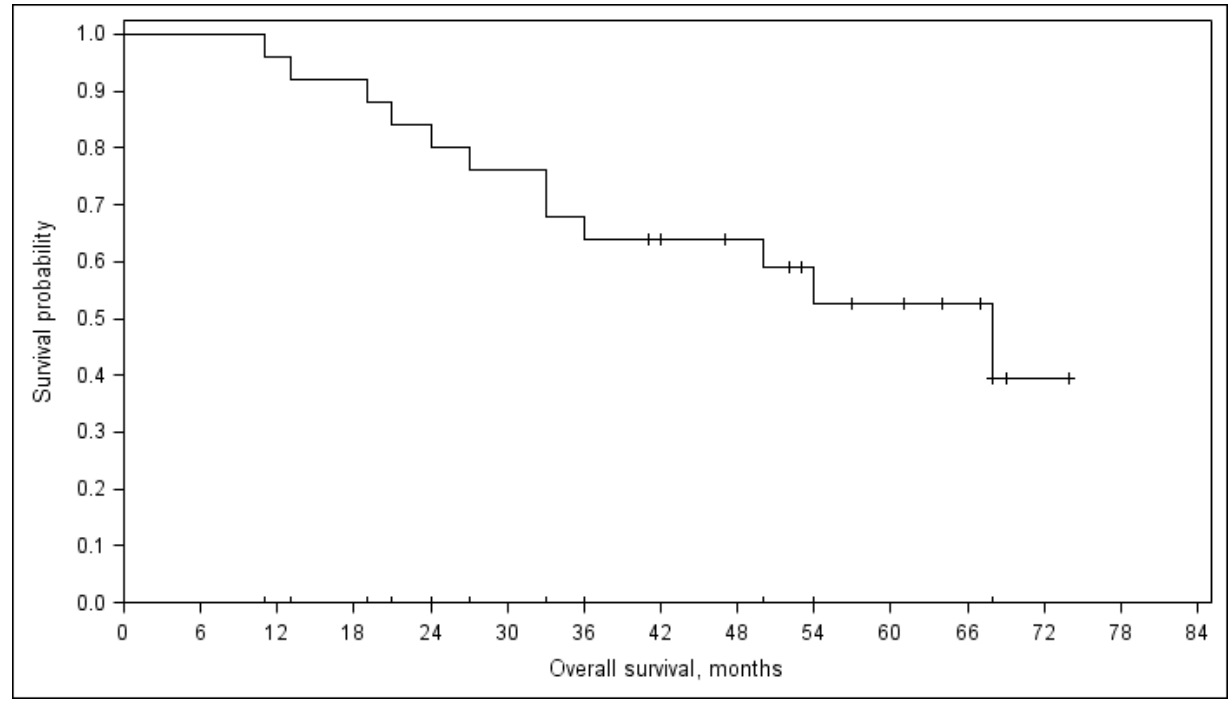

Figure 3. Overall survival (OS).

\subsection{Prognostic Factors for PFS and OS}

Gender, bulky lymphadenopathy, 17p deletion and/or TP53 mutation, IgVH mutational status, number of study treatment courses, ZAP mutations, CD 38 expression, elevated beta- 2 microglobulin, and CD20 expression were evaluated in Cox regression analysis for PFS and OS (Table 2). Univariate analysis showed that 17p deletion/TP53 mutation, bulky lymphadenopathy, positive ZAP70 expression, unmutated $\mathrm{IgVH}$, and increased $\beta_{2}$ microglobulin were predictive of shorter PFS. High CD20 expression $(>90 \%)$ was predictive of longer PFS in univariate analysis. Multivariate analysis confirmed the presence of $17 \mathrm{p}$ deletion/TP53 mutation, unmutated $\operatorname{IgVH}$, and high $\beta_{2}$ microglobulin as adverse factors for PFS. There was a trend of shorter OS in patients with 17p deletion/TP53 mutation and higher $\beta_{2}$ microglobulin.

Table 2. Univariate Cox regression analysis of impact of different prognostic factors on PFS and OS.

\begin{tabular}{ccccccc}
\hline Characteristics & \multicolumn{3}{c}{ PFS } & \multicolumn{3}{c}{ OS } \\
\cline { 2 - 7 } & HR & $\mathbf{9 5 \% ~ C I ~}$ & $p$ & HR & $\mathbf{9 5 \% ~ C I ~}$ & $p$ \\
\hline Male gender & 0.840 & $0.319-2.208$ & 0.723 & 1.117 & $0.333-3.750$ & 0.858 \\
p17del/TP53 mutation * & 6.314 & $1.905-20.930$ & 0.003 & 3.285 & $0.952-11.337$ & 0.060 \\
Cycles No $\leq 4$ vs. $>4$ & 1.060 & $0.448-2.507$ & 0.895 & 0.344 & $0.074-1.598$ & 0.174 \\
Bulky disease $>5$ cm & 2.847 & $1.140-7.114$ & 0.025 & 2.081 & $0.656-6.607$ & 0.214 \\
ZAP expression & 3.850 & $1.343-11.039$ & 0.012 & 1.392 & $0.418-4.641$ & 0.590 \\
IgVH mutation * & 0.168 & $0.051-0.548$ & 0.003 & 0.319 & $0.070-1.466$ & 0.142 \\
CD38 expression $\geq 20 \%$ & 1.070 & $0.469-2.440$ & 0.872 & 0.595 & $0.189-1.877$ & 0.376 \\
CD20 expression > 90\% & 0.976 & $0.956-0.996$ & 0.019 & 0.987 & $0.960-1.014$ & 0.332 \\
$\mathrm{~B}_{2}$ microglobulin $>3.5 \mathrm{mg} / \mathrm{L} *$ & 1.303 & $1.045-1.625$ & 0.019 & 1.228 & $0.999-1.510$ & 0.051 \\
\hline
\end{tabular}

* Independent predictive factors for PFS in multivariate Cox regression.

\subsection{Toxicity}

Clinically significant adverse events were recorded during treatment and up to three months after the last treatment cycle (Table 3). AEs were mainly grade I-II ( $81 \%)$ and consisted primarily of cardiovascular events (hypertension, supraventricular extrasystolia, atrial fibrillation) and hypokalemia and infections (upper respiratory tract infections, urine tract infection, herpes labialis). Hypokalemia was transient, related to HDMP administration, and was corrected by potassium supplements without clinically significant relevant sequelae. Hematologic toxicity was limited and consisted primarily of neutropenia. Seven patients experienced 16 episodes of grade III-IV neutropenia. Three cases of febrile 
neutropenia were observed. G-CSF was administered in five (20\%) patients. There were no deaths during the treatment period. Grade III-IV infections were noted in three patients (12\%). The HDMP dose was not reduced for any patient.

Table 3. Adverse events.

\begin{tabular}{|c|c|c|c|}
\hline Adverse Events & $\begin{array}{l}\text { Grade I/II } \\
\text { n (\%) }\end{array}$ & $\begin{array}{c}\text { Grade III/IV } \\
\text { n (\%) }\end{array}$ & $\begin{array}{c}\text { Subjects with at Least One AE } \\
\mathbf{n}\end{array}$ \\
\hline All reported & $106(81)$ & $25(19)$ & $x x x$ \\
\hline Chills & $1(9.4)$ & & 1 \\
\hline Cardiovascular disturbances & $17(16.0)$ & $1(4.0)$ & 15 \\
\hline Gastrointestinal disturbances & $4(3.8)$ & & 3 \\
\hline Hematology & & $4(16.0)$ & 4 \\
\hline Neutropenia & $2(1.9)$ & * $16(64.0)$ & 7 \\
\hline Infections & $11(10.4)$ & $4(16.0)$ & 8 \\
\hline Urticaria papulosa & $1(9.4)$ & & 1 \\
\hline Lumbalgia subacuta & $3(2.8)$ & & 2 \\
\hline Arthralgias/headache & $4(3.8)$ & & 4 \\
\hline Cough & $3(2.8)$ & & 3 \\
\hline Hyperuricemia & $1(9.4)$ & & 1 \\
\hline Renal insufficiency & $2(1.9)$ & & 2 \\
\hline Thrombosis & $2(1.9)$ & & 2 \\
\hline Fever & $5(4.7)$ & & 3 \\
\hline Edema & $5(4.7)$ & & 4 \\
\hline Left femur cervical stress fracture & $1(9.4)$ & & 1 \\
\hline Cognitive disturbance & $1(9.4)$ & & 1 \\
\hline Hyperglycemia & $10(9.4)$ & $1(4.0)$ & 2 \\
\hline Hypokalemia & $30(28.3)$ & & 18 \\
\hline Insomnia & $3(2.8)$ & & 3 \\
\hline
\end{tabular}

\subsection{Salvage Therapy}

Twenty-two patients received salvage treatment after the study. The median time to first treatment was 19 (3-46) months. Eight (36\%) of 22 LT-CLL-2s patients received ibrutinib as salvage treatment. Median (range) OS from first post-study relapse treatment was 17 (4-30) months in chemo (immuno) therapy and $44(41-47)$ months in the ibrutinib group $(p=0.085)$. There was a trend of better survival for patients given ibrutinib at any point during disease progression (Figure 4).

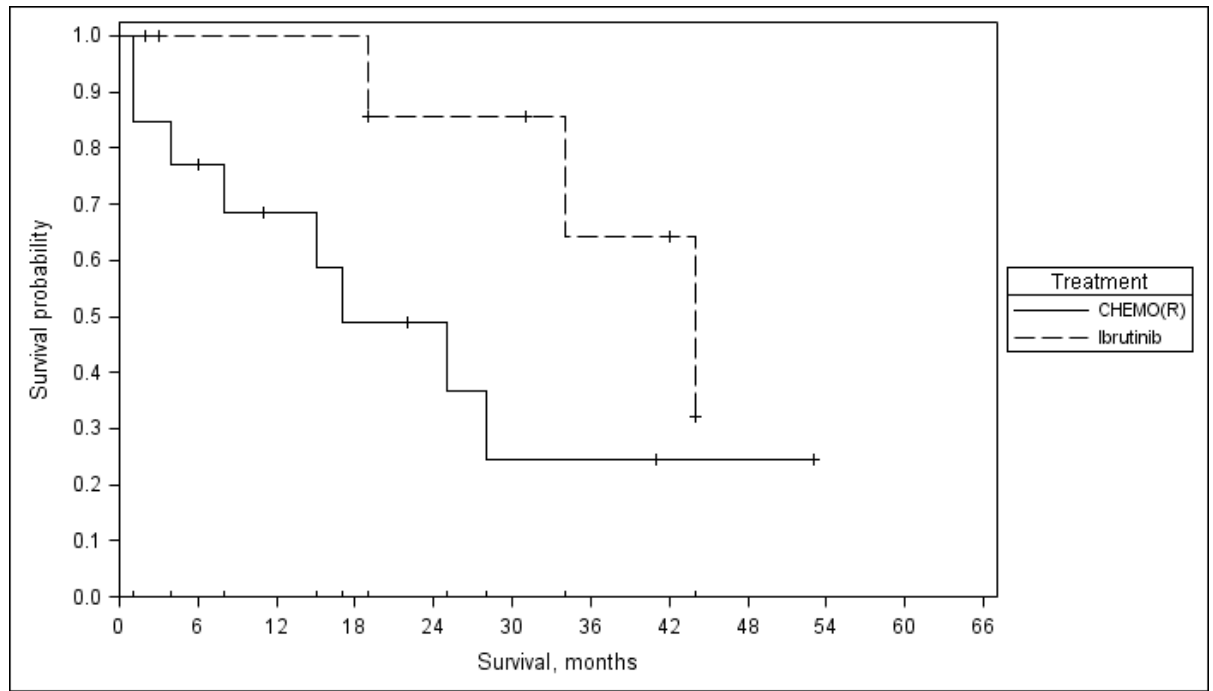

Figure 4. Overall survival from first salvage according to given treatment. 


\section{Discussion}

Before the era of pathway inhibitors, monoclonal anti-CD20 antibodies and high-dose glucocorticoid combinations were evaluated in poor-prognosis patients (Table 4).

Table 4. Summary of high-dose glucocorticoid and antiCD20 antibodies combination treatment in the literature.

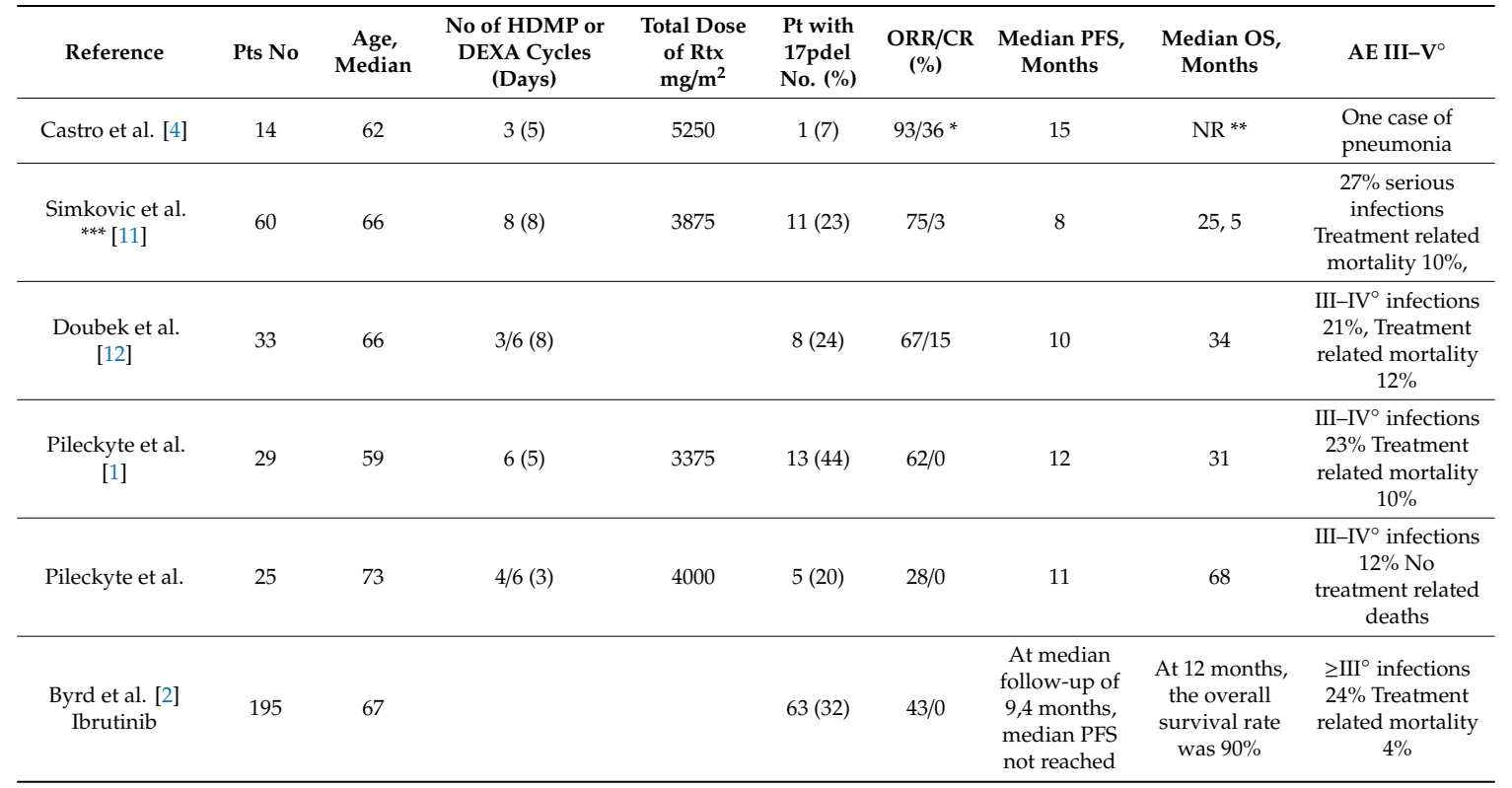

* CT scans not performed for response evaluation. ${ }^{* *}$ Not reached after a median follow up of 40 months.

*** Retrospective study.

Castro et al. [4] treated 14 refractory or fludarabine-intolerant patients with HDMP-Rtx and achieved an ORR of $93 \%$ and a CR of $36 \%$ with favorable safety profiles. In this study, the highest cumulative Rtx dose was applied, however, only three patients had a poor prognosis and CT scans were not included in the response evaluation.

In our previous study [3], we evaluated a Rtx/HDMP combination for relapsed high-risk patients. Higher HDMP dose per treatment course $\left(5 \mathrm{~g} / \mathrm{m}^{2}\right.$ vs. $\left.3 \mathrm{~g} / \mathrm{m}^{2}\right)$ and slightly lower Rtx dose per complete treatment period $\left(3375 \mathrm{mg} / \mathrm{m}^{2} \mathrm{vs} .4000 \mathrm{mg} / \mathrm{m}^{2}\right.$ ) was used in the LT-CLL-001 study compared to the LT-CLL-2s study (Table 4). The median age was 59 years old vs. 73 years old, and there were more high-risk patients in LT-CLL-001 vs. LT-CLL-2s: TP53 mut/17p del $44 \%$ vs. $20 \%$, IgHV unmutated, $86 \%$ vs. $68 \%$, fludarabine refractory $34 \%$ vs. $8 \%$. The ORR was $62 \%$ (all PRs) vs. $28 \%$ (all PRs), PFS was 12 vs. 11 months, OS was 31 vs. 68 months, median follow-up was 31 vs. 50 months, and there were three vs. zero treatment-related deaths in the LT-CLL-001 vs. LT-CLL-2s study, respectively. Nine patients (31\%) were $\geq 65$ years of age in the LT-CLL-001 trial and seven $(78 \%)$ responded to this treatment. The median PFS in this patient group was 13 months and the median OS was 40 months. There was one death during the treatment period of an elderly patient due to gastrointestinal bleeding.

Eight (36\%) of LT-CLL-2s patients received ibrutinib vs. one (5\%) of 20 LT-CLL-001 patients as salvage, which could have contributed to longer OS in the LT-CLL-2s study.

Simkovic et al. [11] presented retrospective data of Rtx and high-dose dexamethasone combination with an ORR of $75 \%$, median PFS of 8 months, and median OS of 25.5 months. ORR was not dependent on age, but in multivariate analysis, an age of $\geq 65$ years and an absence of therapeutic response (SD/PD) were identified as independent predictors of shorter PFS ( $p=0.002$ for both). Only an age of $\geq 65$ years was a significant predictor of shorter OS $(p=0.006)$, which may be explained by higher treatment toxicity in the elderly population. 
Doubek et al. [12] evaluated 33 relapsed refractory CLL patients treated with at least three cycles of an ofatumumab and high-dose dexamethasone combination. ORR was $67 \%$, the median PFS was 10 months, and the median OS was 34 months.

Smolej et al. [13] ran the largest prospective study, combining high-dose dexamethasone with two schedules of high-dose Rtx $\left(1500 \mathrm{mg} / \mathrm{m}^{2}\right.$ on days $1,8,15,22$ with $375 \mathrm{mg} / \mathrm{m}^{2}$ in the first dose, repeated every 4 weeks (group I) and $2500 \mathrm{mg} / \mathrm{m}^{2}$ on day one $\left(375 \mathrm{mg} / \mathrm{m}^{2}\right.$ in first cycle) repeated every 3 weeks (group II)), evaluated 54 relapsed or refractory (R/R) CLL patients with ORR of $62-72 \%$ and a high CR rate of $21 \%$ in patients receiving a lower dose of Rtx but also having a lower incidence of bulky disease. Response evaluation did not include bone marrow examination, and imaging methods included CT scans or ultrasound examination. Median follow-up was less than 12 months with a median PFS of 6-9 months and a median OS of 14 months (group I) vs. not reached (group II). Serious infections occurred in $32 \%$ of the patients, resulting in three early deaths.

Overall, the ORR was more than $60 \%$ in these studies and the median PFS was 8-12 months. The ORR in our study was only $28 \%$, largely due to residual lymphadenopathy confirmed by CT imaging. However, our patients achieved the median PFS of 11 months, which is comparable to the previous studies. In the LT-CLL-001 study, the interim analysis at three months and after the end of therapy [3] demonstrated increasing hemoglobin and platelet levels, as well as a $17 \%$ improvement in lymph node response. These observations suggest that longer exposure to high doses of gliucocorticoids translates into a better response and may influence progression-free survival but may also cause higher toxicity.

Grade III-IV infections were diagnosed in $21-27 \%$ of cases and the treatment-related mortality was $10-12 \%$ in glucocorticoid/Rtx combination studies (Table 4). Notably, grade III-IV infections were also observed in $24 \%$, and $4 \%$ of patients died in the Ibrutinib arm of the RESONATE trial [2] (Table 4).

None of the previous trials were specifically designed for the elderly, relapsed patient population which is known to be more fragile and susceptible to infectious complications. In our study, III-IV infections were observed in $12 \%$ of patients. Importantly, there were no treatment-related deaths (Table 4).

The longer median OS in the LT-CLL-2s study (68 months) compared to the LT-CLL001 study (31 months) may have been due to the lower number of high-risk patients (TP53 mut $/ 17 \mathrm{p}$ del $20 \%$ vs. $44 \%$, IgHV unmutated, $68 \%$ vs. $86 \%$, fludarabine refractory $8 \%$ vs. $34 \%$ ), the higher availability for BTK inhibitors as salvage therapy ( $36 \%$ vs. $5 \%$ of patients), and a significantly lower toxicity (treatment related mortality $0 \%$ vs. 10\%) in the LT-CLL-2s study. This was achieved despite the LT-CLL-2s patients being older than those in the LT-CLL-001 study (median age 73 vs. 59 years, respectively).

\section{Conclusions}

In conclusion, when targeted therapies are unavailable or contraindicated, a HDMP and Rtx combination may be applied either as a 5-day HDMP regimen in relapsed refractory high-risk cases or a 3-day regimen in elderly or unfit CLL patients. Further studies of HDMP and Rtx combination in CLL refractory to pathway inhibitors are warranted.

Author Contributions: R.P. performed the study, supervised the study, evaluated the results and wrote the manuscript. V.V., M.S. performed the genetic analysis. R.M. performed flow cytometry. J.S. described CT scans. T.Z. performed statistical analysis. L.G. designed the study, wrote the protocol, performed the study, supervised the study, evaluated the results and wrote the manuscript.

Funding: This research received no external funding.

Conflicts of Interest: R.P., L.G.: research support from Roche Lietuva was achieved (study drug Rituximab was provided by Roche Lietuva) V.V., M.S., J.S., R.M., A.J., T.Z. have no conflicts-of-interest to disclose.

\section{References}

1. Eichhorst, B.; Hallek, M.; Goede, V. Management of unfit elderly patients with chronic lymphocytic leukemia. Eur. J. Intern. Med. 2018, 58, 7-13. [CrossRef] [PubMed] 
2. Byrd, J.C.; Brown, J.R.; O’Brien, J.C.; Barrientos, N.E.; Kay, N.M.; Reddy, S.; Coutre, C.S.; Tam, S.P.; Mulligan, U.; Jaeger, S.; et al. Ibrutinib versus Ofatumumab in Previously Treated Chronic Lymphoid Leukemia. N. Engl. J. Med. 2014, 371, 213-223. [CrossRef] [PubMed]

3. Pileckyte, R.; Jurgutis, M.; Valceck, V.; Stockus, M.; Gineikene, E.; Sejoniene, J.; Degulys, A.; Zvirblis, T.; Griskevicius, L. Dose-dense high-dose methylprednisolone and rituximab in the treatment of relapsed or refractory high-risk chronic lymphocytic leukemia. Leuk. Lymphoma 2011, 52, 1055-1065. [CrossRef] [PubMed]

4. Castro, J.E.; Sandoval-Sus, J.D.; Bole, J.; Rassenti, L.; Kipps, T.J. Rituximab in combination with high-dose methylprednisolone for the treatment of fludarabine refractory high-risk chronic lymphocytic leukemia. Leukemia 2008, 22, 2048-2053. [CrossRef] [PubMed]

5. Bowen, D.A.; Call, T.G.; Jenkins, G.D.; Zent, C.S.; Schwager, S.M.; Van Dyke, D.L.; Jelinek, D.F.; Kay, N.E.; Shanafelt, T.D. Methylprednisolone-rituximab is an effective salvage therapy for patients with relapsed chronic lymphocytic leukemia including those with unfavorable cytogenetic features. Leuk. Lymphoma 2007, 48, 2412-2417. [CrossRef] [PubMed]

6. Hallek, M.; Cheson, B.D.; Catovsky, D.; Caligaris-Cappio, F.; Dighiero, G.; Döhner, H.; Hillme, P.; Keating, M.J.; Montserrat, E.; Rai, K.R.; et al. Guidelines for the diagnosis and treatment of chronic lymphocytic leukemia: A report from the International Workshop on Chronic Lymphocytic Leukemia updating the National Cancer Institute-Working Group 1996 guidelines. Blood 2008, 111, 5446-5456. [CrossRef] [PubMed]

7. Bastien, R.; Lewis, T.B.; Hawkes, J.E.; Quackenbush, J.F.; Robbins, T.C.; Palazzo, J.; Perou, C.M.; Bernard, P.S. High-throughput amplicon scanning of the TP53 gene in breast cancer using high-resolution fluorescent melting curve analyses and automatic mutation calling. Hum. Mutat. 2008, 29, 757-764. [CrossRef] [PubMed]

8. Van Dongen, J.J.; Langerak, A.W.; Bruggemann, M.; Evans, P.A.; Hummel, M.; Lavender, F.L.; Delabesse, E.; Davi, F.; Schuuring, E.; Garcia-Sanz, R.; et al. Design and standardization of PCR primers and protocols for detection of clonal immunoglobulin and T-cell receptor gene recombinations in suspect lymphoproliferations: Report of the BIOMED-2 Concerted Action BMH4-CT98-3936. Leukemia 2003, 17, 2257-2317. [CrossRef] [PubMed]

9. Salvi, F.; Miller, M.D.; Grilli, A.; Giorgi, R.; Towers, A.L.; Morichi, V.; Spazzafumo, L.; Mancinelli, L.; Espinosa, E.; Rappeli, A.; et al. A manual of guidelines to score the modified Cumulative Illness Rating Scale andits validation in acute hospitalized elderly patients. J. Am. Geriatr. Soc. 2008, 56, 1926-1931. [CrossRef] [PubMed]

10. Common Terminology Criteria for Adverse Events v4.03 (CTCAE). Available online: http://evs.nci.nih.gov/ ftp1/CTCAE/CTCAE_4.03_2010-06-14_QuickReference_5x7.pdf (accessed on 14 June 2010).

11. Šimkovič, M.; Motyčková, M.; Belada, D.; Vodárek, P.; Kapoor, R.; Jaffar, H.; Vrbacký, F.; Žák, P.; Smolej, L. Five years of experience with rituximab plus high-dose dexamethasone for relapsed/refractory chronic lymphocytic leukemia. Arch. Med. Sci. 2016, 12, 421-427. [CrossRef] [PubMed]

12. Doubek, M.; Brychtova, Y.; Panovska, A.; Sebejova, L.; Stehlikova, O.; Chovancova, J.; Malcikova, J.; Smardova, J.; Plevova, K.; Volfova, P.; et al. Ofatumumab Added to Dexamethasone in Patients with Relapsed or Refractory Chronic Lymphocytic Leukemia. Results from a Phase II Study. Am. J. Hematol. 2015, 90, 417-421. [CrossRef] [PubMed]

13. Smolej, L.; Doube, M.; Panovskac, A.; Simkovi, M.; Brychtovab, Y.; Belada, D.; Motyckova, M.; Mayer, J. Rituximab in combination with high-dose dexamethasone for the treatment of relapsed/refractory chronic lymphocytic leukemija. Leuk. Res. 2012, 36, 1278-1282. [CrossRef] [PubMed]

(C) 2019 by the authors. Licensee MDPI, Basel, Switzerland. This article is an open access article distributed under the terms and conditions of the Creative Commons Attribution (CC BY) license (http://creativecommons.org/licenses/by/4.0/). 